\title{
3D-Simulation Data-Making Trial to present and analyze Small-sized Farmlands Fields with Car-shaped Robot, ROS2, SLAM and Foxy for Real agricultural workers
}

\author{
Shinji Kawakura and Ryosuke Shibasaki
}

\begin{abstract}
In this study, we create various application systems focusing on agricultural (agri-) field data digitalization issues that will benefit traditional agri-researchers, workers, and their respective managers. We obtain three-dimensional (3D) information on agri-environments (e.g., rice fields, farmlands) via roaming robots with sensors. Robot-controlled middleware, such as robot operating systems (ROS), are often used for such robots. Thus, we selected car-shaped robot (NANO-RT1), ROS2, and the SLAM-based system. The carshaped robot-based system operates sensor units uniformly. With this technology, we can recognize our location at an unknown place, and the robot can run. There are challenges in accurately presenting quantitative accuracy data for this type of study. We address this by providing average and standard deviation (SD) data for certain situations using five algorithms: (1) Hector-SLAM, (2) G-mapping, (3) Karto-SLAM, (4) CoreSLAM, and (5) Lago-SLAM. We believe the proposed holistic system has the potential to improve not only agri-businesses, but also agri-skills and overall security levels.
\end{abstract}

Keywrods - 3D-Simulation data-making, car-shaped robot, NANO-RT1 Robot, ROS2, SLAM, Foxy, agricultural site.

\section{INTRODUCTION}

Over several decades, various hardware agricultural (agri) systems have been developed to help manage agri-business [1]-[11].

Obtaining and sharing diverse digital three-dimensional (3D) agri-field information is a crucial factor for success in practical modern agri-management. One approach to achieving this is to use an autonomous mobile robot. Thus, we develop a robot using simultaneous localization and mapping (SLAM) systems.

When developing such self-controlled locomotive robots, researchers and engineers often utilize middleware of robot control as robot operating systems (ROS) or the more advanced ROS2 [12]-[21].

In this study, considering past technical concepts and trends, we develop and implement a ROS2 SLAM-based agri-fields' record producing system for common agriworkers and managers.

Specifically, we use the car-shaped NANO-RT1 Robot (Shenzhen XiaoR Geek Technology Co. Ltd., Shenzhen, China), which can integrate and handle diverse sensors and electric modules. This allowed us to execute these trials with

Submitted on November 09, 2021.

Published on November 30, 2021.

S. Kawakura, Research Center for Artificial Photosynthesis (ReCAP) at Osaka City University, Japan.

(corresponding e-mail: s.kawakura@gmail.com) an assimilation, to some extent.

\section{METHOD}

\section{A. Outline}

First, we reviewed past academic and industry novel accomplishments and met with predecessors who have engaged in primary industries. Then, we selected promising ROS2 and SLAM-based techniques. Next, we developed approximate schedules, designed, and mechanically constructed the systems, and performed various processing.

Although diverse similar systems and commercial goods exist, none are optimized for small- to middle-sized, nontrimmed (i.e., not well prepared for running car-shaped machines) agri-fields with 3D-SLAM systems or cooperate with network-connected systems. Thus, we create new integrated structures that support these options and enhance their utility and flexibility against sudden accidents in real situations.

We implemented certain distributed smartphone applications on the car-shaped NANO-RT1 Robot. As preliminary trials, we conducted practical experiments to judge the systems' utility in an indoor (sufficiently flat home) situation and an outdoor asphalt road.

Thinking of recent technical trends [12]-[21], we considered the following five algorithm-based SLAM approaches to compare diverse items, particularly error estimation value: (1) Hector-SLAM, (2) G-mapping, (3) Karto-SLAM, (4) Core-SLAM, and (5) Lago-SLAM.

The following main features of the SLAM framework for this study were considered as criteria for selection: (a) sensors' installation; (b) data gathering mechanism, including an odometer; (c) algorithm; (d) mapping methods; and (e) looping back directions.

\section{B. System}

Fig. 1-3 illustrate the main structure of our approach and proposed system. Fig. 1 shows the fundamental flow of the system's methodology, Fig. 2 illustrates the model of the proposed ROS 2 car robot, and Fig. 3 shows the set of systems used to achieve SLAM-based operations.

This study consists of five phases: (1) designing and confirming the validity of the entire system; (2) constructing and integrating mechanical parts, including tuning and

R. Shibasaki, Center for Spatial Information Science (CSIS), The University of Tokyo, Japan.

(e-mail: shiba@ csis.u-tokyo.ac.jp) 
revising minor system settings; (3) conducting preliminary experiments in both indoor and outdoor settings; (4) conducting main trials in outdoor farmlands; and (5) evaluating output datasets with consideration to similar research.

We mounted a Raspberry Pi (Raspberry Pi Foundation, Cambridge, England (UK)) and a Jetson Nano (Nvidia Corporation Inc., California, USA) as the microcomputer board. We flexibly coded in both Python and $\mathrm{C}++$ languages.

The NANO-RT1 was $300 \times 240 @ 325 \mathrm{~mm}$, weighing $3.1 \mathrm{~kg}$. The main camera had 1,080 pixels. The Inertial Measurement Unit (IMU) was a nine-axis gyroscope sensor. The driver board was STM32F105. The motor torque was $3.5 \mathrm{~N} \cdot \mathrm{m}$. Finally, the radar was XR_Lidar S1.

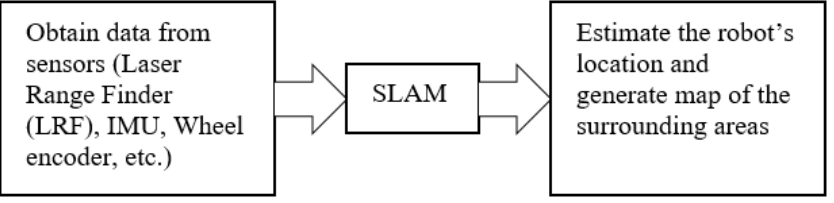

Fig. 1. Fundamental flow of this system's methodology.

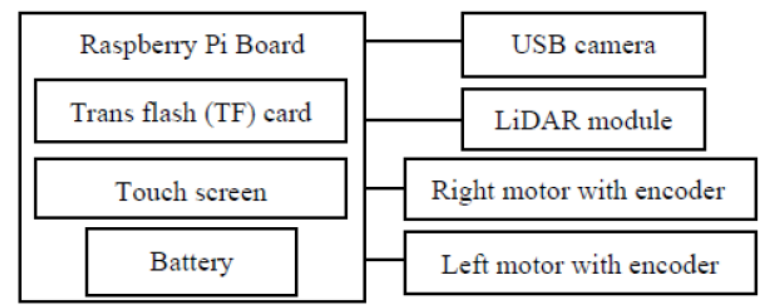

Fig. 2. Model of the Raspberry Pi-based ROS2 car-shaped robot.

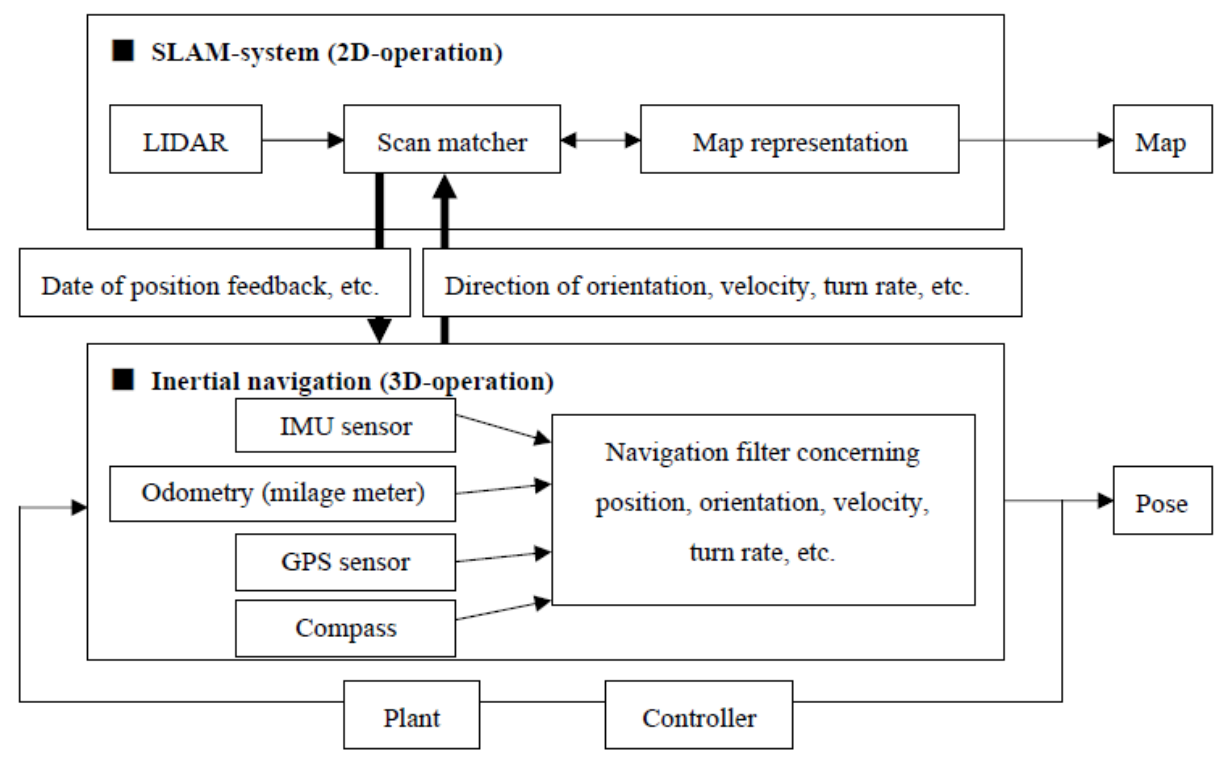

Fig. 3. Set of systems for SLAM-based operations.

Fig. 4-7 illustrate the real parts of the study, i.e., the actual built components.

Fig. 4 presents the distributed smartphone application software used to build electronic maps. Fig. 5 shows the Ubuntu operating system (OS) version 18.04 installed on the Raspberry Pi board. Fig. 6-7 present the deep learning-based car-shaped machines that we tested through successive studies.

Fig. 8 shows a simplified map of the target agri-field. The gray areas are a common, non-specific, stiff plastic-sheet road (corridor) that we placed to reduce the physical barriers of the car-shaped robot's movement.

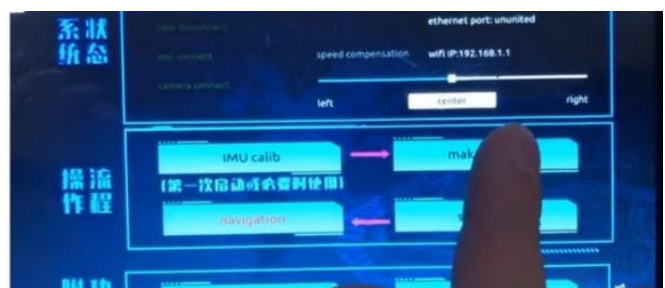

Fig. 4. Distributed smartphone application to build electronic maps.

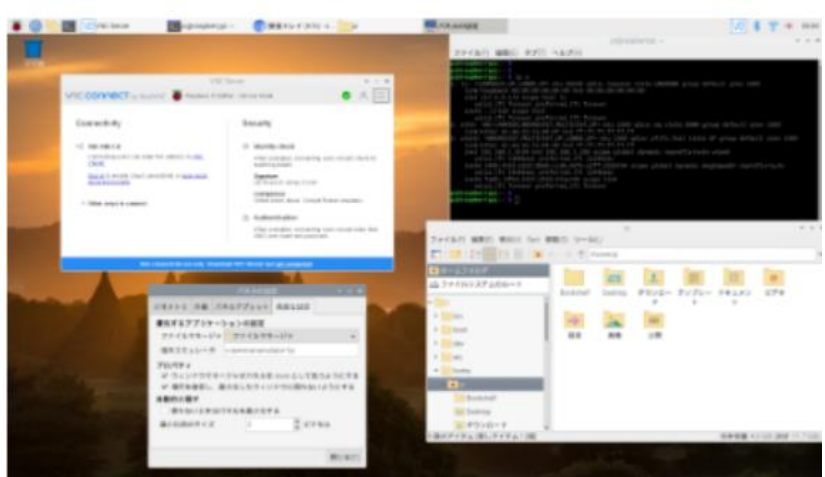

Fig. 5. Setting of Ubuntu OS ver. 18.04 on Raspberry Pi board.

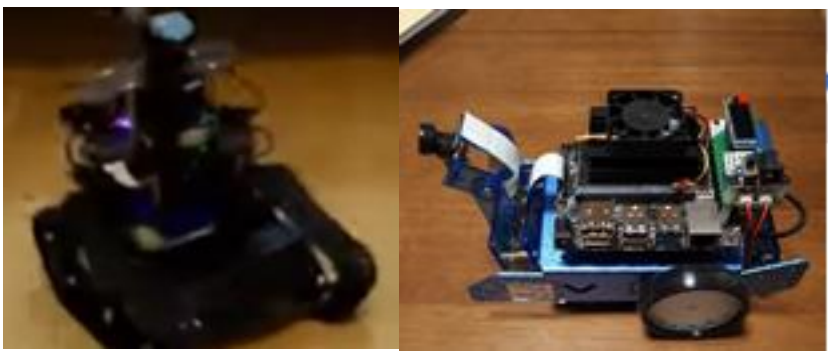

Figs. 6-7. Deep learning-based car-shaped machines that the authors tested through successive studies. 


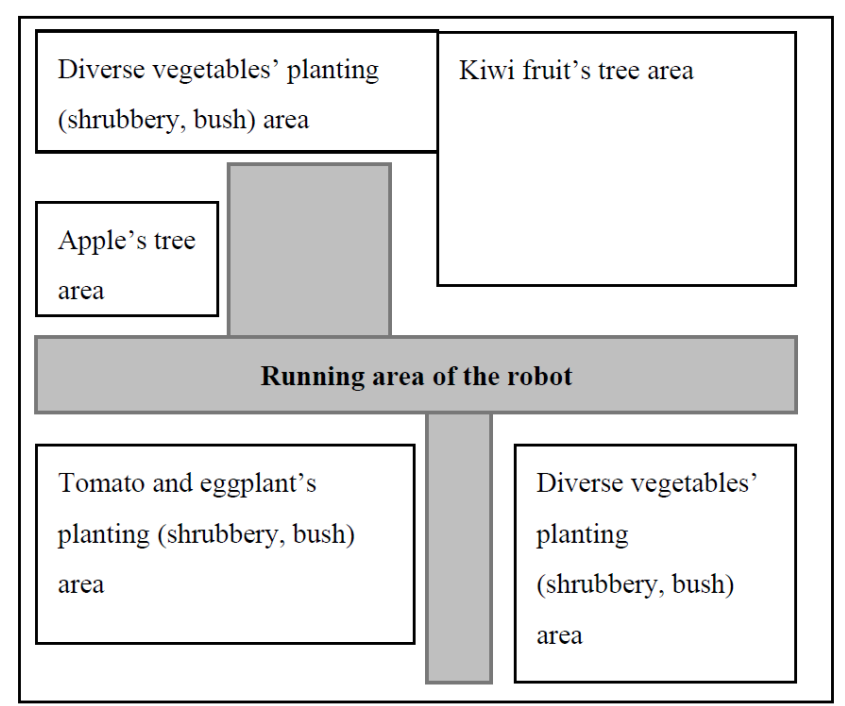

Fig. 8. Simplified map of target agri-field.

\section{Theory}

We compared the usefulness and characteristics of five algorithms: (1) Hector-SLAM, (2) G-mapping, (3) KartoSLAM, (4) Core-SLAM, and (5) Lago-SLAM.

Here, note that Hector-SLAM's navigation technologies are sometimes combined with other two-dimensional (2D) SLAM methodologies for better robustness and inertial sensing.

Note that 3D spatial navigation state estimation is based on Extended Kalman Filter (EKF), which is only necessary when the IMU exists. Thus, we only discuss 2D SLAM in this study.
We initially obtained 2D map data in target fields to set the laser beam lattice (vertical position) and estimate the representation of laser points on the mapping data. Then, we assumed the probability (\%) of matching each squared grid.

In this study, we used a Gauss-Newton-based methodology to resolve the scan-matching data and identify the rigid body transformation of the laser pointer to set the current map to existing map data.

We performed scan matching by finding the rigid transformation presented in (1).

$$
\xi=(x, y, \theta)^{T}=\left(p_{x}, p_{x}, \Psi\right)^{T}
$$

We then aim to minimize $\xi$ as in (2)

$$
\xi^{*}=\operatorname{argmin}(\xi)=\sum_{i=1}^{n}\|1-M(\operatorname{Si}(\xi))\|^{2}
$$

In Equation (2), $\operatorname{Si}(\xi)$ is a global coordinate of the $i-$ th scan endpoint $s_{i}=\left(s_{i, x}-s_{i, y}\right)^{T}$, and $M(S i(\xi))$ returns the map value for these coordinates. The problem is solved using the Gauss-Newton equation:

$$
\Delta \xi=H^{-1}[\nabla M(\operatorname{Si}(\xi))]^{2}
$$

The Hessian Matrix $H$ is given by (4)

$H=\left[\nabla M(\operatorname{Si}(\xi)) \frac{\partial \operatorname{Si}(\xi)}{\partial \xi}\right]^{T} \cdot\left[\nabla M(\operatorname{Si}(\xi)) \frac{\partial \operatorname{Si}(\xi)}{\partial \xi}\right]$

Fig. 9 presents the methodology of the ROS2-based system.

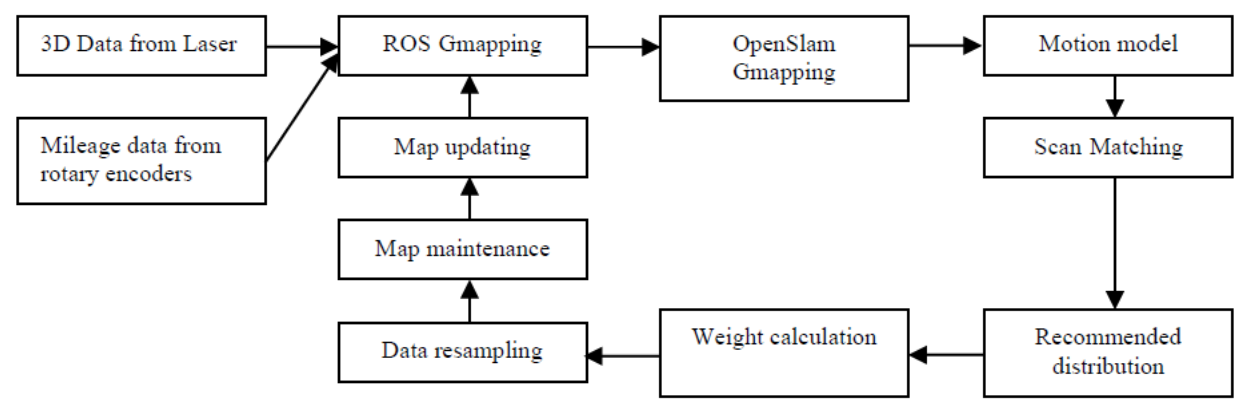

Fig. 9. ROS2 system

\section{RESULTS}

As described, we created a SLAM system with a carshaped robot that included sensors to realize ROS2.

Fig. 10 presents the error-estimation data for the five SLAM-based approaches, where the bars indicate the average values with standard deviations. The number of trials $(\mathrm{N})$ is five.

Fig. 11-12 present typical sample pictures of ROS2 operations. The arrow-shaped marks indicate the location of the robot.

Based on our results, the proposed approaches could realize moderate accuracy compared with similar approaches found in the literature and industry. However, we could not quantify the difference between our proposed system and past similar systems using specific statistical items. We did not define appropriate trial time ranges; the trial area was rather narrow. Qualitatively, we confirmed the usefulness and feasibility of the proposed system.

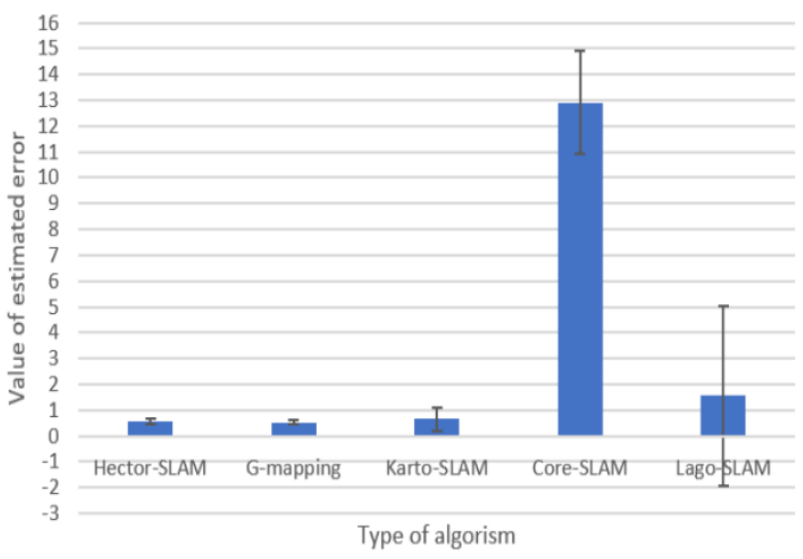

Fig. 10. Comparative graph of average error estimation for 5 SLAM algorithms with standard deviation whiskers $(\mathrm{N}=5)$. 


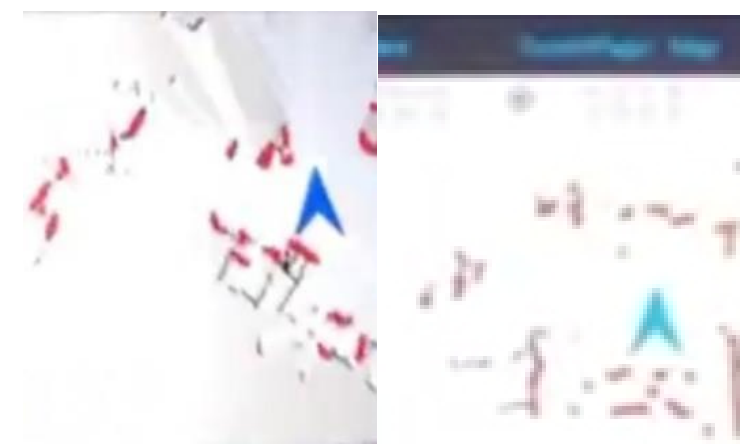

Figs. 11-12. 3D data from prototype ROS2 system.

\section{DISCUSSION AND CONCLUSION}

To gather ambient, agri-situation 3D data, we accumulated basic data from several digital methodologies with the aim of future practical applications. We calculated and compared various patterns using five SLAM-based algorithms: (1) Hector-SLAM, (2) G-mapping, (3) Karto-SLAM, (4) CoreSLAM, and (5) Lago-SLAM.

However, the agility, cost-performance, and usefulness of the proposed approach is promising and could easily be applied by agri-managers or agri-researchers.

As we could not present item-oriented (scientifically numerical) sets of fixed quantitative data, we must seek more suitable and valid methods to assess the systems' operations.

Another remaining problem is attaining more data from diverse settings and situations (such as breeding farms, plastic farms, construction sites, or in situations with more intense or sudden natural impacts).

Our desktop analyses and interviews with key experts and users could be incorporated into computer systems and fed back to experimental users.

We could also undertake further experiments and practical applications of the system to understand how to enhance the system for use in real settings.

\section{FUTURE TASKS}

For future scalabilities, we have been planning consultation and supporting projects to improve the security and productivity of agri-workers. Above all, we hope to start launching practical discussions, consultations, and proposals with real agri-users, both in Japan and globally.

The techniques we have examined are economically robust, scalable, and distributed flexibly. Therefore, they could be applied to other fields and settings, such as factories or residential districts, to identify sudden accidents or crimes and to solve diverse social problems.

In the future, we will attach small solar panels to the robot's body to support long-term operation.

Additionally, as part of troubleshooting the system's limitations, we will consider various non-fixed factors, such as utilizing locations, and sudden incidents, such as the system's tumbling or sudden halting.

\section{ACKNOWLEDGMENT}

Our heartfelt appreciation goes to the members of Mitsui Fudosan Co., Ltd., Kashiwano-Ha Farm Inc., Kashiwa-shi, National Agriculture and Food Research Organization
(NARO, Tsukuba-shi, Japan), and The University of Tokyo who provided considered support, feedback, and comments.

\section{REFERENCES}

[1] S. Zhao, Z. Zhang, D. Xiao, and K. Xiao. A Turning Model of Agricultural Robot Based on Acceleration Sensor. IFAC-Papers OnLine, vol. 49, no. 16, pp. 445-450, Jan. 2016.

[2] Y. Funami, S. Kawakura, and K. Tadano. Development of a Robotic Arm for Automated Harvesting of Asparagus. European Journal of Agriculture and Food Sciences, vol. 2, no. 1, pp. 1-10, Jan. 2020.

[3] S. Rivera. Securing Robots: An Integrated Approach for Security Challenges and Monitoring for the Robotic Operating System. Doctoral dissertation at University of Luxembourg, Luxembourg, May 2021.

[4] M. Cebe, E. Erdin, K. Akkaya, H. Aksu, and S. Uluagac. Block4forensic: An integrated lightweight blockchain framework for forensics applications of connected vehicles. IEEE Communications Magazine, vol. 56, no. 10, pp. 50-57, Oct. 2018.

[5] G. Marques, and R. Pitarma. Agricultural environment monitoring system using wireless sensor networks and IoT. Proceedings of IEEE 2018 13th Iberian Conference on Information Systems and Technologies (CISTI), pp. 1-6, June 2018.

[6] M. Cebe, E. Erdin, K. Akkaya, H. Aksu, and S. Uluagac. Block4forensic: An integrated lightweight blockchain framework for forensics applications of connected vehicles. IEEE Communications Magazine, vol. 56, no. 10, pp. 50-57, Oct. 2018.

[7] A. Zujevs, V. Osadcuks, and P. Ahrendt. Trends in robotic sensor technologies for fruit harvesting: 2010-2015. Procedia Computer Science, vol. 77, pp. 227-233, Jan. 2015.

[8] R. Vidoni, R. Gallo, G. Ristorto, G. Carabin, F. Mazzetto, L. Scalera, and A. Gasparetto. An Agricultural Mobile Robot Prototype for Proximal Sensing and Precision Farming. Proceedings of ASME (American Society of Mechanical Engineers) 2017 International Mechanical Engineering Congress and Exhibition, no. V04AT05A057, pp. 1-7, Jan. 2017.

[9] V. F. Tejada, M. F. Stoelen, K. Kusnierek, N. Heiberg, and A. Korsaeth. Proof-of-concept robot platform for exploring automated harvesting of sugar snap peas. Precision Agriculture, vol. 18, no. 6, pp. 952-972, April 2017.

[10] L. N. Smith, W. Zhang, M. F.Hansen, I. J. Hales, and M. L. Smith. Innovative $3 \mathrm{D}$ and $2 \mathrm{D}$ machine vision methods for analysis of plants and crops in the field. Computers in Industry, vol. 97, pp. 122-131, 2018.

[11] L. Siefke, V. Sommer, B. Wudka, and C. Thomas. Robotic systems of systems based on a decentralized service-oriented architecture. Robotics, vol. 9, no. 78, Sep. 2020.

[12] S. Dragule. Programming Robots for Activities of Everyday Life. Doctoral dissertation at Department of Computer Science and Engineering, Chalmers University of Technology, 2021.

[13] V. Mayoral, A. Hernández, R. Kojcev, I. Muguruza, I. Zamalloa, A. Bilbao, and L. Usategi. The shift in the robotics paradigm-The Hardware Robot Operating System (H-ROS); an infrastructure to create interoperable robot components. Proceedings of IEEE of NASA/ESA Conference on Adaptive Hardware and Systems (AHS), pp. 229-236, July 2017.

[14] E. A. Esquivel-Barboza, L. F. Llamas, P. Vázquez, F. Bellas, and E. Arias-Méndez. Adapting Computer Vision Algorithms to Smartphonebased Robot for Education. Proceedings of Fourth International Conference on Multimedia Computing, Networking and Applications (MCNA) 2020, pp. 51-56, Oct. 2020.

[15] V. Strobel, E. Castelló Ferrer, and M. Dorigo. Managing byzantine robots via blockchain technology in a swarm robotics collective decision-making scenario. Proceedings of the 17th International Conference on Autonomous Agents and Multi Agent Systems, pp. 541549, July 2018.

[16] R. Shamshiri, I. Hameed, et. al. Simulation software and virtual environments for acceleration of agricultural robotics: Features highlights and performance comparison. Int. J. Agric. \& Biol. Eng., vol. 11, no.4, pp. 15-31, April 2018.

[17] G. Lumer-Klabbers, J. O. Hausted, J. L. Kvistgaard, H. D. Macedo, M. Frasheri, and P. G. Larsen. Towards a Digital Twin Framework for Autonomous Robots. Proceedings of 2021 IEEE 45th Annual Computers, Software, and Applications Conference (COMPSAC), pp. 1254-1259, July 2021.

[18] M. Pantano, T. Kamps, S. Pizzocaro, G. Pantano, M. Corno, and S Savaresi. Methodology for Plant Specific Cultivation through a Plant Identification pipeline. Proceedings of 2020 IEEE International 
Workshop on Metrology for Agriculture and Forestry (MetroAgriFor), pp. 298-302, Nov.2020.

[19] R. Cale. Modeling and Control of a Four-wheel Autonomous Agricultural Robot. Master's thesis at NTNU, 2021.

[20] G. Deng, Y. Zhou, Y. Xu, T. Zhang, and Y. Liu. An Investigation of Byzantine Threats in Multi-Robot Systems. Proceedings of 24th International Symposium on Research in Attacks, Intrusions and Defenses, pp. 17-32, Oct. 2021.

[21] S. Macenski and I. Jambrecic. SLAM Toolbox: SLAM for the dynamic world. Journal of Open Source Software, vol. 6, no. 61, pp. 1-7, May 2021.

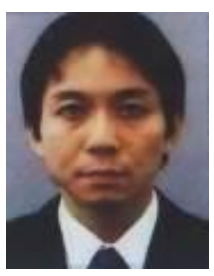

Shinji Kawakura was born on July 14, 1978 in Toyama Pref., Japan. He received Ph.D. in Environmentology, University of Tokyo in 2015 Bunkyo-ku, Tokyo, Japan; B.A. in Control System Engineering, Tokyo Institute of Technology in 2003, Meguro-ku, Tokyo, Japan; M.A. in Human-Factor Engineering, Tokyo Institute of Technology in 2005, Meguro-ku, Tokyo, Japan.

Career: Systems engineering, research for private companies. Development and verification of sensing systems for outdoor agricultural workers.

Dr. Kawakura, Research Center for Artificial Photosynthesis (ReCAP) at Osaka City University/Osaka City, Osaka, Japan. IEEE senior member, Hong Kong Chemical, Biological \& Environmental Engineering Society (HKCBEES) senior member.

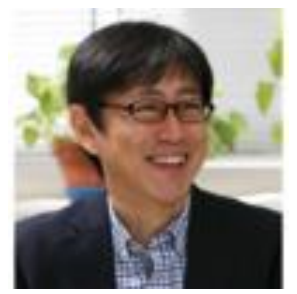

Ryosuke Shibasaki was born on March 1, 1958 in Fukuoka. Pref. Japan. R. Shibasaki received Dr. in Engineering, the University of Tokyo in 1987, Bunkyo-ku, Tokyo, Japan; B.A in Engineering, the University of Tokyo in 1980, Bunkyo-ku, Tokyo, Japan; M.A. in Engineering, the University of Tokyo in 1982, Bunkyo-ku, Tokyo, Japan.

Career: Professor at the Center for Spatial Information Science, University of Tokyo.

Dr. Shibasaki, Center for Spatial Information Science (CSIS), The University of Tokyo, Meguro-ku, Tokyo, Japan, and Department of SocioCultural and Socio-Physical Environmental Studies, The University of Tokyo/Kashiwa-shi, Chiba, Japan. 